\title{
The Three Stages of Radar Plotting
}

\author{
Captain H. Topley
}

MANY discussions have taken place regarding the feasibility of plotting when using radar in reduced visibility and it soon becomes apparent that there is confusion in what each of us considers by the term 'plotting'.

These notes are to suggest definitions of the various stages of plotting and to consider the conditions under which each is necessary.

Radar plotting can be divided into three stages:

(a) Record plotting

(b) Deductive plotting

(c) Forecast plotting.

\section{Record Plotting}

This is the recording of three or more radar ranges and bearings on a suitable plotting surface. By far the easiest way of doing this is on a reflection plotter if one is provided. If not provided with a reflection plotter then it must be done on a spider web as a relative plot, or on a sheet of paper or chart as a true plot.

A minimum of three ranges and bearings is necessary to determine whether the other vessel has maintained her course and speed during the plotting interval. This record plotting is necessary as it is not possible just from studying the ranges and bearings to determine whether they lie on a straight line evenly spaced with regard to time.

If this plotting has been done on a relative plot it will indicate at once whether there is risk of collision and, when in congested waters, it will probably be all the plotting required on the great majority of the targets since it will show at that time that there is no risk of collision with them.

If this record plotting can be done on a reflection plotter, particularly if the set is being used with a relative display then a large number of echoes can be recorded with comparative ease. If no reflection plotter is available then the time required to transfer the ranges and bearings to a plotting sheet will very much reduce the number of target echoes which can be handled by a single observer.

\section{Deductive Plotting}

This is the next stage and in general is only necessary when there is risk of collision, and it is required to find the approximate courses and speeds of the targets to determine the most suitable avoiding action within the terms of the recommendation appended to the new Collision Regulations.

If the record plotting has been done using a true-motion display or has been done on a true plot then deductive plotting is generally necessary on a considerable proportion of the echoes to determine whether there is a real risk of collision or not.

If the record plotting has been done as a relative plot only those targets which have been determined to be risks will need this stage. It need not be particularly accurate. A first estimate of their courses and speeds will usually be sufficient if given approximately as this information is generally, at this stage, only required 
within fairly wide limits, i.e., courses within $20^{\circ}$ to $30^{\circ}$ or so and speeds as 'fast', 'average' or 'slow'.

\section{Forecast Plotting}

This stage is not reached until it has been decided what avoiding action is best. In deciding what action is best again we must consider the recommendation appended to the new Collision Regulations which state that any alteration of course or alteration of speed should be substantial and in neither case should a number of small alterations be made.

This last stage of plotting is to forecast the anticipated future relative movement of the target echo so that its actual movement may be watched to determine whether the target has made any alterations which would cancel out the effect of your own avoiding action. Forecast plotting is also necessary on a selection of near echoes (if any) which were not previously collision risks, to determine whether own ship's alteration might produce a close quarters situation with one or more of them.

In either of these cases if the original risk has not been reduced or a close quarters situation seems likely to arise with another nearby ship then it would be necessary to comply with Rule 16(c) and stop engines in proper time to avoid collision.

In this stage of plotting a slightly higher degree of accuracy is required if early appreciation of the other vessel's action (if any) is to be obtained. In general, however, it is not necessary to work to the degree of accuracy which would be required in an answer to an examination question.

This stage of plotting, apart from the necessity to check nearby echoes for collision risk by deductive plotting, is not necessary if a true display is in use or if the record plotting has been done on a true plot as in this type of plot the forecast is a straight continuation of the target movement if she does not make any alteration.

\section{Conclusions}

Plotting is a three stage exercise and even if only the first stage, record plotting, is done on any particular echo then the observer can be considered to be plotting.

If a relative plot is used then this stage may be all that is necessary in any particular case and if a reflection plotter is available the number of echoes which can be dealt with by a single observer is a maximum.

If a true-motion plot is being used then in most cases deductive plotting will be necessary, and this will reduce the maximum which a single observer can cope with. Against this must be balanced the fact that the third stage, forecast plotting, is not necessary if a true-motion display or a true plot has been used. The second stage deductive plotting may be necessary to determine whether your alteration has produced a collision risk with another vessel which has previously been considered safe. 